\title{
Two approaches for automating analysis of electromagnetic processes in non-linear circuits with valves
}

\author{
Khushnud Sapaev ${ }^{1}$ and Shukhrat Umarov ${ }^{2 *}$ \\ ${ }^{1}$ Technical Sciences Doctor, Professor, Tashkent State Technical University named after I. Karimov, Tashkent, Uzbekistan \\ ${ }^{2}$ Technical Sciences Candidate, Assistant Professor, Tashkent State Technical University named after I. Karimov, Tashkent, \\ Uzbekistan
}

\begin{abstract}
Annotation: This article analyses and compares two approaches related to automating modeling of valve electric circuits with piecewise linear approximation of valve characteristics. The first approach is based on operator equivalent circuits' analytical formulas and on analytical expressions programming which describe equivalent circuits on each conduction interval of valve elements. The second approach provides implementation of a system for modeling valve converters based on implicit numerical integration formulas.
\end{abstract}

Keywords: converter, power supply, mathematical model, operator method, transient process, rectifier, inverter, loading.

As far as is known, the confident solutions of various problems concerning analysis and parametric synthesis of valve converters are associated with the implementation of a large volume of calculations. In this connection, the following should be noted that a significant part of failures related to electronic equipment occurs because of circuit and design flaws that arise mainly due to the use of manual methods for calculating circuits in engineering practice. This is due to the fact that manual calculation methods are based, as a rule, on a number of simplified assumptions that significantly reduce the quality of design and suggest the need for experimental prototyping - a very timeconsuming and expensive process. Therefore, a widespread use of digital computers (DC) in the design process is a prerequisite for improving the quality, efficiency and shortening the development time of valve converters.

The modern technological development horizon of computer technology allows us to raise the question of not only performing separate calculations, but it also has made it possible to determine the optimal parameters of circuit components, to simulate various dynamic modes, to explore any combination of circuit elements, and thereby provides automation of design and development work related to the implementation into production of various complex schemes of valve converters.

The greater part of programs for analyzing electrical circuits with valves use valve element models idealized on the basis of a number of assumptions, which are practicality confirmed by practice. In this case, the current-voltage characteristics (CVC) of essentially nonlinear elements - valves correspond to the characteristics of an ideal key or have a different form, reflecting a piecewise linear approximation (PLA). The PLA of nonlinear characteristics and the use of digital computers for calculations allow widespread use in the analysis of circuits the fitting method for the intervals of valve conductivity [1], in which the electric circuit is considered as a linear on the segments between the break points of the approximated characteristics, and the structure or parameters of the circuit change when moving from one segment to another.

Possible ways to fitting method implementation on a digital computer (DC) may be:

- The program development based on the classical methods of transient analysis;

- The creation and use of universal programs, including models of electric circuits power elements and their control systems.

A correlative study of the two approaches which has been described above is carried out on the example of calculating a parallel autonomous current inverter $[1,2$, 3] with a compensating device. Schematic diagram of a stand-alone current inverter (SACI) with a compensating device $(\mathrm{CD})$ is shown in the Fig. 1.

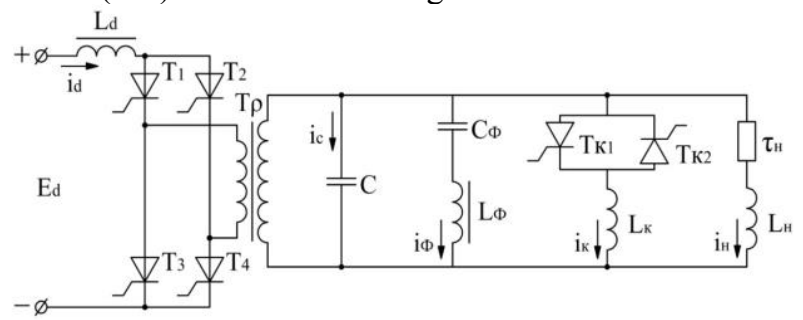

Fig. 1. Parallel SACI with CD Schematic Diagram.

\footnotetext{
* Corresponding author: shumarov1951@mail.ru
} 
The circuit operate main valves are in operation in pairs (T1, T4 - T2, T3). They do switch at a frequency of $50 \mathrm{~Hz}$. The pair T1 - T4 is at the initial moment in a conducting state. Phase shift between the output voltage $u_{H}$ and the output current of the inverter $i_{H}$ is $\beta_{\beta}=45^{\circ}$. The compensating device, consisting of the elements $L \kappa$, $R \kappa, T 5, T 6$, is turned on for the sixth period with an angle $\alpha=29^{\circ}$, counted to the left along the time axis from the transition of the voltage curve through "Zero".

Initial approximation to the circuit analysis machine implementation has been based on analytical formulas of operator equivalent circuits and programming of analytical expressions describing equivalent circuits on each conduction interval of valve elements whose characteristics correspond to ideal keys [4, 5, 6]. Also programmable is the key state management algorithm. The mathematical models development is to be carried out in the following sequence:

a) Many possible types of equivalent circuits are to be determined. That will occur with the selected method of excitation and stabilization;

b) The equivalent universal circuits are to be compiled. Current images and originals and, also, required voltages in the calculation are to be displayed;

c) Different possible ways of the process development are to be analyzed and the sequence of equivalent circuits changing types at the clock intervals will be revealed. The boundary conditions for the process development stages change are to be formed this way;

d) An algorithm block diagram for calculating the transient will be compiled this way. The algorithm block diagram is based on the search for the fulfillment of boundary conditions taking into account the selected method of excitation and regulation.

The analysis of possible operator equivalent circuits (OECs) shows that a complete calculation of the transient process of parallel SACI with $\mathrm{CD}$ may be carried out on the basis of two universal OECs that differ only in operating state of CD. One of them corresponds to the switched-off state; the other corresponds to switched-on state. Their using for the analysis of various operational situations is determined by the corresponding initial conditions. The calculation formulas of instantaneous values of the sought currents and voltages are to be derived for each of these universal OECs [3].

The currents and voltages originals are to be expressed as:

$$
\begin{gathered}
i_{d}(t)=\tau_{1} E_{d} / a_{12}+\left(B_{2} / D_{1}\right) \exp \left(-6_{2} t\right)+ \\
+\left(B_{3} / D_{2}\right) \exp \left(-6_{3} t\right)+\mathrm{A}_{1} \mathrm{~K}_{1}+\mathrm{B}_{1} \mathrm{~K}_{2} ; \\
u_{c}(t)=\tau_{H} \tau_{\mathrm{K}} E_{d} / \mathrm{a}_{12}+\left(B_{5} / D_{1}\right) \exp \left(-6_{2} t\right)+ \\
+\left(B_{6} / D_{2}\right) \exp \left(-6_{3} t\right)+\mathrm{A}_{4} \mathrm{~K}_{1}+\mathrm{B}_{4} \mathrm{~K}_{2} ; \\
i_{\mathrm{K}}(t)=\tau_{H} E_{d} / \mathrm{a}_{12}+\left(B_{5} / D_{3}\right) \exp \left(-6_{2} t\right)+ \\
+\left(B_{6} / D_{4}\right) \exp \left(-6_{3} t\right)+\mathrm{A}_{4} \mathrm{~K}_{3}+\mathrm{B}_{4} \mathrm{~K}_{4} ; \\
i_{\mathrm{H}}(t)=\tau_{H} E_{d} / \mathrm{a}_{12}+\left(B_{5} / D_{5}\right) \exp \left(-6_{2} t\right)+ \\
+\left(B_{6} / D_{6}\right) \exp \left(-6_{3} t\right)+\mathrm{A}_{4} \mathrm{~K}_{5}+\mathrm{B}_{4} \mathrm{~K}_{6} ;
\end{gathered}
$$

The obtained analytical expressions have been programmed and a process development algorithm has been determined. In result we obtained a mathematical model, with the help of which transient processes were calculated, and sought currents and voltages time diagrams were constructed, shown in Fig. 2, 3 and 4.

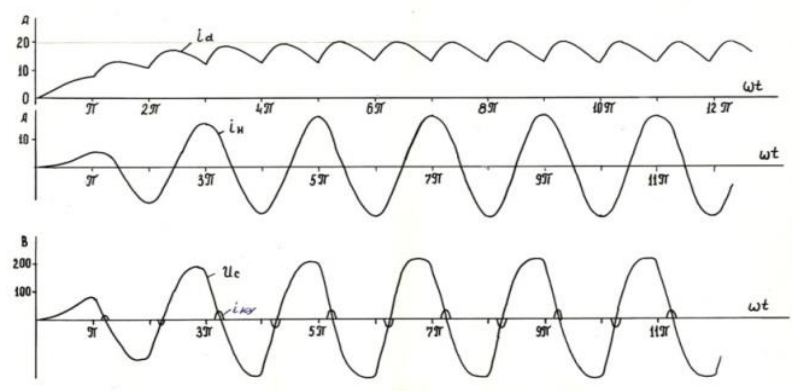

Fig. 2. Currents and Voltages Timing Diagrams at Start-up

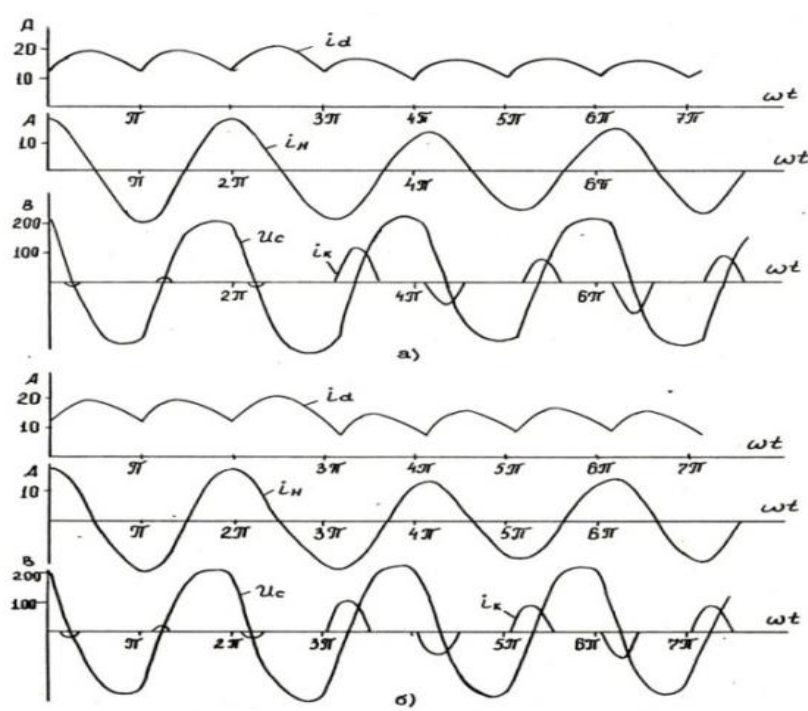

Fig. 3. Currents and Voltages Timing Diagrams during Load Shedding: a) Independent Excitation; b) Combined Excitation.
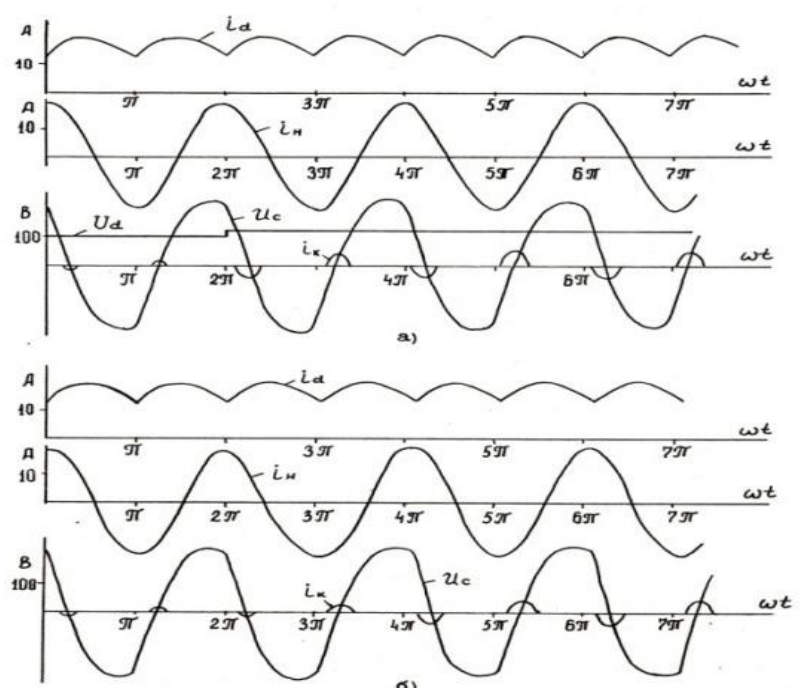

Fig. 4. Currents and Voltages Timing Diagrams during Input Voltage Increase: a) Independent Excitation; b) Combined Excitation. 
We have undertaken a series of computer studies using the developed mathematical model (algorithm and program). The recommended parameters and part types of the SIP circuit elements based on a parallel inverter operating in a continuous mode of the input current of the inverter were obtained.

The second approach is implemented using a simulation system of valve converters based on implicit numerical integration formulas (PSIs) [7], while the valve characteristics $\mathrm{VC}$ of the valves take into account on-state losses (differential resistance $r_{d}=0.001 \mathrm{Ohm}$, threshold voltage $\left.\mathrm{u}_{0}=0.1 \mathrm{~B}\right)$. The initial data prepared during the statement of the problem carry information about the circuit corresponding to one initial state of the valve elements. In the process of machine solution, after each switching of valves, a new equivalent circuit of the simulated circuit is automatically formed. The state of the valves is controlled using the developed algorithm. The minimum integration step is assumed to be $2 \mu \mathrm{s}$. The maximum value of the step automatically obtained during the operation of self-accelerating PSIs is $2150 \mu \mathrm{s}$.

A comparison of two approaches to automating analysis showed that the discrepancy in the results does not exceed tenths of a percent. The error was estimated by instantaneous values (the values were obtained by the analytical method and taken as a standard). It is calculated by the formula:

$$
\varepsilon=\left[\frac{\mathrm{a}-\mathrm{x}}{\mathrm{a}}\right],
$$

Here $\boldsymbol{a}$ is the value obtained by the analytical method, and $\boldsymbol{x}$ is the value obtained in the process of numerical integration.

Table 1. Calculation Selected Results

\begin{tabular}{|c|c|c|c|c|c|c|}
\hline $\begin{array}{c}\text { Time } \\
\text { max. }\end{array}$ & $\begin{array}{c}u_{H} \\
(\text { standard) } \\
\mathrm{B}\end{array}$ & $\begin{array}{c}u_{H} \\
(\mathrm{PSIs}) \\
\mathrm{B}\end{array}$ & $\begin{array}{c}\varepsilon \\
\%\end{array}$ & $\begin{array}{c}i_{H} \\
(\text { standard } \\
\mathrm{A}\end{array}$ & $\begin{array}{c}i_{H} \\
(\text { PSIs) } \\
\mathrm{A}\end{array}$ & $\begin{array}{c}\varepsilon \\
\%\end{array}$ \\
\hline 1667 & 4,287 & 4,279 & 0,19 & 2,085 & 2,082 & 0,19 \\
\hline 3333 & 15,90 & 15,88 & 0,13 & 3,993 & 3,985 & 0,20 \\
\hline$\ldots$ & $\ldots$ & $\ldots$ & $\ldots$ & $\ldots$ & $\ldots$ & $\ldots$ \\
\hline 61667 & $-70,38$ & $-70,24$ & 0,19 & 17,9 & 17,88 & 0,11 \\
\hline 63333 & 58,73 & 58,62 & 0,19 & 20,06 & 20,03 & 0,15 \\
\hline$\ldots$ & $\ldots$ & $\ldots$ & $\ldots$ & $\ldots$ & $\ldots$ & $\ldots$ \\
\hline 121667 & 71,17 & 71,04 & 0,18 & 17,98 & 17,96 & 0,11 \\
\hline 123333 & $-58,75$ & $-58,65$ & 0,17 & 20,15 & 20,12 & 0,15 \\
\hline
\end{tabular}

During the calculation process, the error at the integration step has been taken equal to 0.001 . It should be noted that a decrease in this step had a weak effect on the calculation results.

We can observe from the table data, Table 1: coincidence of the results obtained by the first method (standard) and those obtained by implicit integration formulas is high - the relative error of instantaneous values over significant time periods (there were six periods) lies within $1 \%$.

Summarizing the above we can say that, a design engineer may be in a puzzle: what analysis method should be selected in terms of efficiency and reliability? In this connection, we may say that most methods have a certain relationship and complement to each other $[8,9$, 10]. In order to obtain reliable results that ensure the receipt of valve converters technical parameters, ensuring the fulfillment of the requirements of the technical specifications for the design of an object (taking into account the significant non-linearity of valve elements with a variety of control systems), it is advisable to use a combined modeling technique.

\section{References}

1. B.D. Bedford, R.G. Hoft. Princ. of Inv. Circ. (Wiley, New York, 1964)

2. Bose, Bimal K. Modern power elec. and $\mathrm{AC}$ dr. (Prentice Hall PTR, 2002).

3. Rik De Doncker, Duco W.J. Pulle, Andre Veltman. Adv. Elect. Drives: Analysis, Mod., Contr., 474 (Springer, 2011)

4. Sh.B. Umarov Math. mod. for calc. of trans. in stab. PS on the basis of single-phase seq. inv. cur. Jour. "Probl. of energy. and res. saving", Vol 4, 112-115, (2015)

5. Sh.B. Umarov Math. Mod. of Stab. Power Sup. Based On Cur. Inv. //IJARSET: Vol 6, Issue 8, (August 2019)

6. Sh.B. Umarov, I.A. Abdullabekov Alg. of calc. of trans. of the stab. power supp. on the bas. of a singleph. seq. autonom. cur. inv. with freq. reg. Int. sci. journal. "Young scientist", No. 21, 228-232, (2016)

7. Van Bakkoven W. Linear Imp. Diff. For. of Var. Step and Order// JEEE Frans. On CAS. Vol 2, 109115, (1975)

8. Yu. Zinin, V.Tereshkin, A.Karamov Trans. of thyr. bridge inv. start-up with freq. doub. Power electronics, No. 5, (2011)

9. S.G.German-Galkin Comp. sim. of semicon. sys. in MATLAB env. Textbook, 320, (2001).

10. D.Tarnapowicz, S.German-Galkin "Ener, optim. of mech. sys. with PMSG", "3rd Int. Conf. on Energy and Env. Prot.”, Vol.46, 1-8, (2018) 